\title{
Zur Architektur der WLB
}

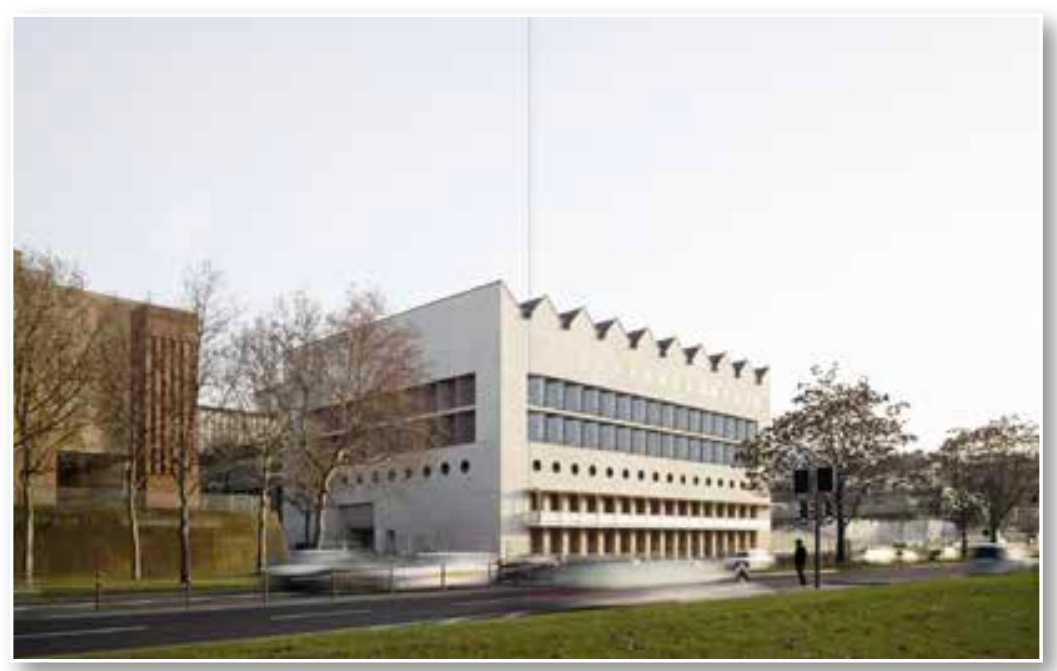

Berlin-Touristen wird das goldene Gehäuse der Staatsbibliothek von Hans Scharoun, schräg gegenüber der Philharmonie, im Gedächtnis bleiben, ohne selbst im Gebäude gewesen zu sein. Sie freuen sich über den Spitznamen "Kommode", der für die ehemalige Universitätsbibliothek am Bebelplatz steht, vergessen darüber gerne, dass das Gebäude schon seit langer Zeit eine andere Nutzung hat. Und wer einmal ein Bild der neuen Universitätsbibliothek von den Architekten Herzog und de Meuron in Cottbus gesehen hat,

\section{Zwei Aufgaben}

Bibliotheksbauten haben zwei Aufgaben zu erfüllen. Zuerst natürlich die, Behälter für Medien zu sein. Medien werden gesammelt, archiviert und für die Öffentlichkeit bereitgestellt. Gebäude, die dafür geplant, erstellt und betrieben werden, sollen perfekt die vielfältigen Funktionen erfüllen: von Magazinen über die Studienbereiche, von Flächen für Gruppen und einzelnen Arbeitsplätzen, von Räumen für Vorträge ebenso wie für Ausstellungen, von Verwaltungsräumen und Aufenthaltsbereichen für Besucher und vieles mehr. Dies ist technisch anspruchsvoll hinsichtlich der klimatischen Konditionierung, des Brandschutzes, der Abtragung hoher Lasten und der Sicherheitsbereiche.

In Wikipedia liest man als erstes von einer Dienstleistungseinrichtung, die ihren Benutzern Zugang zu Information vermittle. Im Wesentlichen beschränkt sich hier die Erläuterung des Begriffs auf die Aufgaben, die Organisation und die Geschichte derartiger Einrichtungen. Wenn wir unabhängig davon von Bibliotheken sprechen, tauchen vor unserem geistigen Auge nicht so sehr diese inneren Organisationen und Aufgaben auf, wir sehen uns wahrscheinlich vielmehr Gebäuden gegenüber, die wir als Bibliotheken aus der Literatur kennen oder die wir selbst besucht haben. Dabei werden sich diese Bilder auf der einen Seite in Gebäude unterscheiden, die wir lediglich von außen im Gedächtnis behalten haben und auf der anderen Seite in beeindruckend große Lesesäle, ohne eine Ahnung davon zu haben, wie deren äußere Hülle beschaffen ist.

\section{nicht vergessen.}

Spätestens seit vor 16 Jahren die Herzogin Anna Amalia Bibliothek in Weimar brannte, zählt ihr Innenraum mit den schönen Buchregalen zum Allgemeinwissen. Die großen Lesesäle der British Library oder der Bibliothèque Nationale de France - Richelieu sind beeindruckende Architekturen (Interieurs), die uns die Frage nach dem heutigen Primat von Funktion und Ökonomie stellen.

Das nämlich ist die zweite, elementar wichtige Aufgabe der Bibliothek: mehr zu sein als ein Behälter für Medien, deren Lagerung und Zugänglichkeit. Sollte sich im Bau einer Bibliothek nicht auch die kulturelle und wissenschaftliche Verfasstheit einer Gesellschaft ausdrücken? Gehört dazu auch der Standort, die bevorzugte Lage in den Innenstädten? Oder umgekehrt: Welcher Verlust drohte, wenn aus Gründen der Funktionen und der Ökonomie die Bibliotheken außerhalb der Stadtzentren ihren Platz fänden? Bräuchte es für solche hochfunktionellen Gebäude mit wenig gestalterischen Anforderungen überhaupt noch Architekten? Kirche, Rathaus, Schule seien die besonderen Gebäude der Stadt, sagt ein Sprichwort aus dem Bereich des Städtebaus. Dazu gehören natürlich auch Büchereien und Bibliotheken. Sie sind geistige und geographische Orientierungspunkte. Wir erkennen sie und ihre Aufgabe im Gewebe der anonymen Bauten. Auch dann, wenn kein Schriftzug an ihnen vorhanden ist, der auf ihre Besonderheit verweist. Orientierung geben diese Häuser auch für die Architektur. Die Öffentliche Hand legt damit den 
Maßstab an, was sie unter Baukultur versteht. Und diese ist es, die unsere Städte lebenswert gestaltet, oder einfacher gesagt, die sie schön macht. Damit also ist die zweite Aufgabe beschrieben, die Bibliotheken zukommt: Ihr Inneres bedingt ein adäquates Äußeres. Hier besteht ein Zusammenhang zwischen der Buchkunst und der Architektur: Das besondere Buch verlangt nach einem besonderen Einband im Unterschied zur Gebrauchsanweisung oder der Alltagsliteratur. Es ist nicht nur an der Machart und der Qualität des Einbandes erkennbar, wir legen es auch an einer hervorgehobenen Stelle ab. (Mag sein, dass diejenigen, die kein Empfinden für ein gutes Buch haben, auch für gute Architektur nicht empfänglich sind).

\section{Die Geschichte als Ideengeber von Architektur und Städtebau}

So jedenfalls dürfte der württembergische König bzw. seine Verwaltung gedacht haben, als sie den Architekten Theodor von Landauer beauftragten, an Stelle des Invalidenhauses in der Neckarstraße 6 , in der die Bibliothek untergebracht war, einen Neubau für die erweiterten Bestände zu erstellen. Interessant an dem zwischen 1875 und 1879 errichteten Gebäude war zum einen die perfekte, fast mechanistische Umsetzung des Raumprogramms, zum anderen die äußere Erscheinung, die weitaus großzügiger gestaltete Innenräume vermuten ließ. Landauer soll sich für die Wiener Weltausstellung begeistert haben, so ist es im Allgemeinen Künstlerlexikon beschrieben. Vielleicht galt Landauers Begeisterung aber eher der Architektur von Gottfried Semper, wie zum Beispiel dem Kunstund Naturhistorischen Museum in Wien - geplant zusammen mit Karl Hasenauer - im Stile der Neorenaissance. Über einem steinernen, die Architektur des Palazzo Pitti zitierenden Sockel erhoben sich überhohe Rundbogenfenster, hinter denen sich nicht etwa große Lesesäle verbargen, sondern eine mehrgeschossige Konstruktion mit Regalen und einzelnen Lesetischen. Auch die Bayerische Hof- und Staatsbibliothek an der Münchner Ludwigstraße, Anfang der 1830er-Jahre in München von Friedrich von Gärtner geplant, zitierte in ihrer Gesamtheit den Palazzo Pitti. Vielleicht schimmerte hinter den Bauten, die nun der Neckarstraße eine Fassung gaben, das prominente Beispiel der Ludwigstraße hervor. Direkt neben dem Neubau stand schon das Naturalien- und Staatsarchiv von Gottlob Georg Barth, ebenfalls, am anderen Ende der Straße, die Staatsgalerie, beide in klassizistischer Architektursprache. Gegenüber der Stuttgarter Bibliothek stand die Hohe Carlsschule, daneben die Reithalle von Salucci, ebenfalls Architekt des Wilhelmspalais und vis-à-vis der Staatsgalerie die Münze. 1912 ergänzte das Operngebäude die schwäbische Version der Ludwigstraße, ein schöner und repräsentativer Stadtraum, der die Innenstadt mit den südöstlichen Wohnquartieren perfekt verband.

Luftaufnahmen vor dem Zweiten Weltkrieg zeigen das Nebeneinander dieser öffentlichen Bauten, selbst nach der Zerstörung war dieser städtebauliche Kontext noch vorhanden. Oper und Reithalle hatten schadlos den Krieg überstanden, die Bibliothek hatte zwar erhebliche Schäden, die Fassaden aber standen noch. Im Bereich zwischen dem Mittelrisalit und der Archivstraße richtete man in den alten Mauern wieder Räumlichkeiten ein. Horst Linde plante den Neubau hinter den Resten des Altbaus, um den Betrieb bis zum Umzug aufrechterhalten zu können. Allerdings hätte er das Volumen auch im Bereich des abgebrochenen Altbaus bis zur Neckarstraße entwickeln können. Die Vorstellung, die Stadt zwischen dem Österreichischen Platz und dem heutigen Gebhard-Müller-

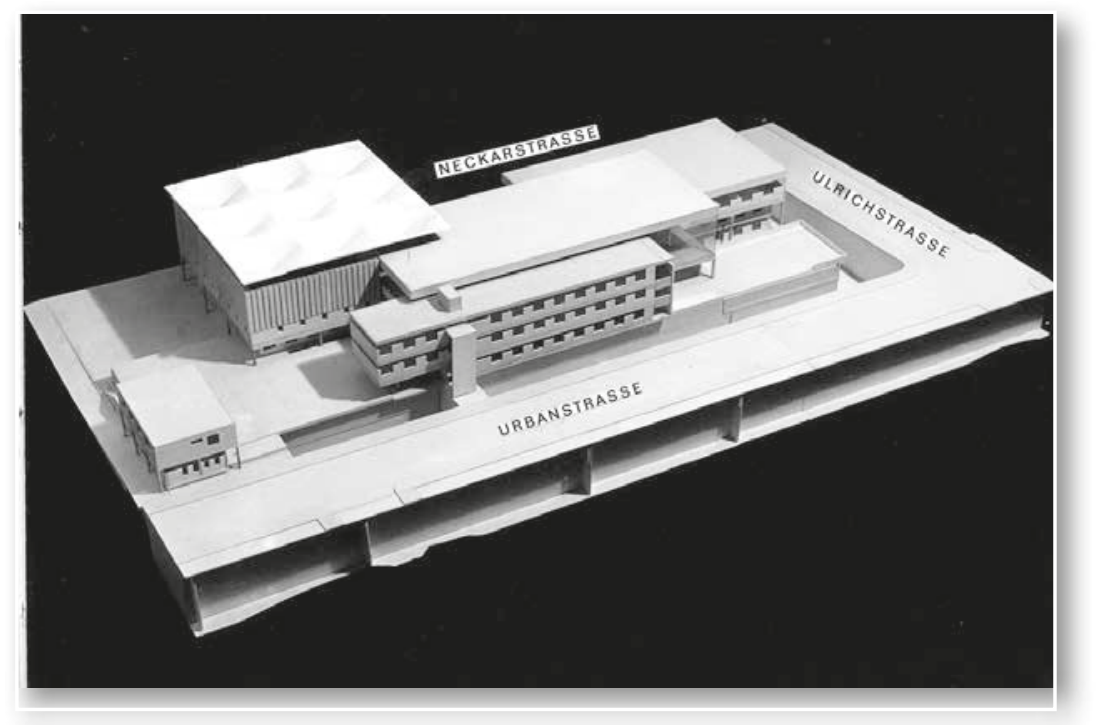

Modell Linde-Neubau

Platz im Sinne der Moderne zu transformieren, war für die rückwärtige Lage ausschlaggebend.

Die Architektur der Moderne kennzeichnet auch den Linde-Bau. Fließende Grundrisse, ein Spiel 
von unterschiedlichen Raumhöhen, teilweise große Glasflächen abwechselnd mit Ziegelwänden, Kupferverkleidung und Sichtbeton: ein durch und durch exzellentes Beispiel der Nachkriegsarchitektur in Deutschland. Auffallend ist das Foyer mit den Lufträumen, der Rampe inklusive Wasserbecken, dem Spiel mit den unterschiedlichen Niveaus und der Materialisierung. Ein räumlich großzügiges Konzept, das heute aus ökonomischen Gründen nicht mehr umsetzbar wäre - ein großer architektonischer und damit verbundener sozialer Verlust. (Merkwürdig fanden wir den Umstand, dass erst durch die Einlassung der Architekten die Bibliothek unter Denkmalschutz gestellt wurde).

\section{Der Erweiterungsbau}

Die Geschichte dieses Ortes, von der stadträumlichen Idee Thourets über den Bau von Landauer zur "zweiten Zerstörung" der Stadt durch den Wiederaufbau bis zur heutigen unbefriedigenden Situation der Verkehrsschneise (die lediglich eine Folge der Vorstellung war, man könne die gewachsene Struktur gegen einen modernen Städtebau z.B. nach der Charta von Athen tauschen) bildet die Grundlage für den Entwurf des neuen Erweiterungsbaus. Weder die Rekonstruktion des alten Stadtraums noch das Weiterbauen im Sinne der Moderne bestimmen die architektonische und städtebauliche Idee. Vielmehr beschäftigte uns die Frage, ob es die Möglichkeit gäbe, für die beiden konträren Vorstellungen von Stadt und Architektur einen dritten Lösungsansatz zu finden.

Pragmatisch kann man es so sehen: Wir bedienen uns der Rochade Lindes in umgekehrter Reihenfolge. Das Neubauvolumen stellen wir vor die heutige Bibliothek, ohne in diese baulich einzugreifen. Damit besetzt der Baukörper teilweise das Baufeld, auf dem das Gebäude Landauers stand. War die ehemalige Neckarstraße bislang durch die Außenwände von Garagenbauwerken begrenzt, erhält sie an dieser Stelle wieder eine bauliche Fassung. So bekommen wir zwischen dem Neubau und dem Staatsarchiv einen neuen urbanen Platz mit hoher Aufenthaltsqualität. Das Volumen des bestehenden Lesesaales wird von diesen beiden Bauten gefasst und steht nun sichtbar in der Mittelachse des Neuen Schlosses. Eine breite Treppenanlage verbindet den neuen Platz mit der Konrad-Adenauer-Straße. Die Bibliothek rückt wieder, für jedermann sichtbar, in die Reihe der wichtigen öffentli- chen Bauten: von der Alten Staatsgalerie bis zum Wilhelmspalais, verbunden durch eine Platanenallee, die den wiedergewonnenen Bürgersteig bis zum

Charlottenplatz begleitet. Auf dem Bibliotheksplatz soll als Spiegelung zu Thourets Brunnen $\longrightarrow$ im Akademiegarten der „Fitz-Faller-Brunnen“ installiert werden, der wegen Stuttgart 21 in den Mittleren Anlagen entfernt wurde. Wir sind dankbar, dass der Kunstausschuss des Landes diesem Vorschlag gefolgt ist. Im Prinzip folgt diese Idee auch unserem Gesamtkonzept, die Wunden der beiden Zerstörungen, vor und nach dem Krieg, zu heilen.

Wir erläutern die Geschichte des Entwurfs von Landauer, das Äußere wie das Innere betreffend, nicht ohne Grund: Das Programm für den Erweiterungsbau ließ wenig Spielraum für großzügige Raumfolgen im Vergleich zum Linde-Bau zu. Im Verlaufe der Überarbeitungen des Wettbewerbsentwurfes wurden geringere Geschoss-

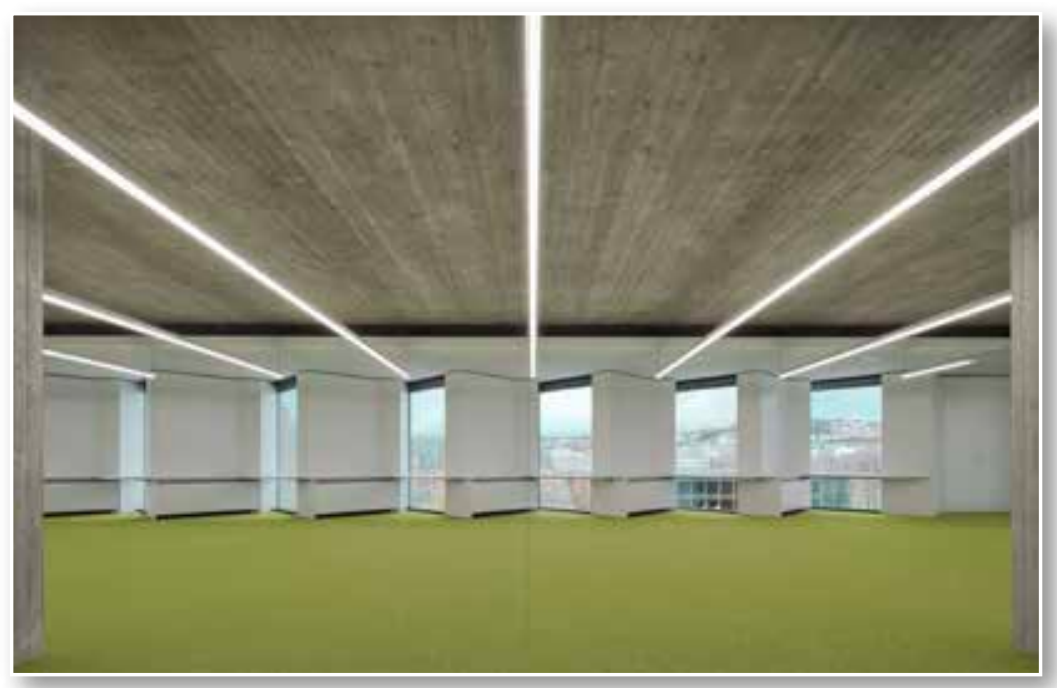




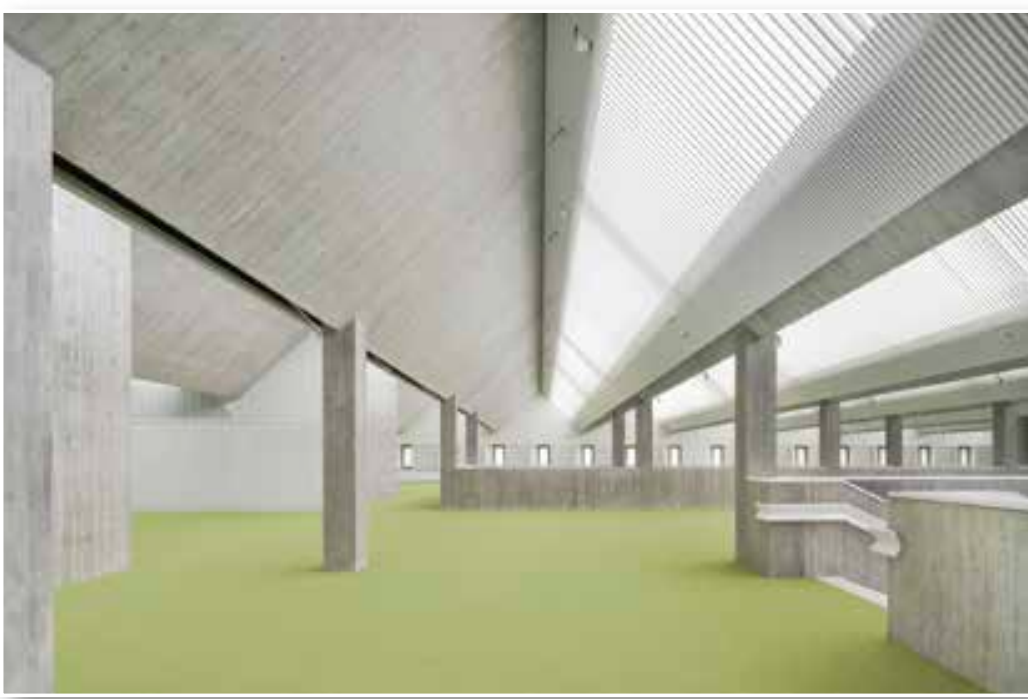

höhen vorgegeben, um Baukosten einzusparen, verbunden mit einer schmerzlichen Einbuße an Raumqualität. Dies wird für den Betrachter von außen nicht sichtbar. Vor allem deshalb, weil die Fensternischen zwei Geschosse verbinden. Auch die beiden Erdgeschosse sind durch die Fassadenstruktur zusammengefasst. Die Materialisierung mit Sichtbeton und einer Kupferverkleidung bezieht sich auf den Altbau und bindet beide Volumen zu einem neuen Ensemble zusammen. Mit dem Erweiterungsbau verknüpft ist für uns der Wunsch, dass die neue Bibliothek die zwei eingangs erwähnten Aufgaben erfüllt: Dass sie nicht nur ein Behälter für Medien ist und ihre vielfältigen Funktionen erfüllt, sondern, dass sie vielmehr auch über ihre Gestaltung und ihre Räume im kollektiven Bewusstsein der Bürger einen Platz findet.

\section{Die Sanierung des Altbaus}

Bei der nunmehr anstehenden Sanierung des Linde-Baus zahlt es sich aus, den Neubau unabhängig vom Bestand errichtet zu haben. Beide Häuser sind durch die Brücke im ersten Obergeschoss verbunden. Diese bleibt noch bis zum Ende der Sanierung geschlossen und kann, ohne weitere Umbaumaßnahmen und ohne zeitlichen Verlust, nach Fertigstellung geöffnet werden. Die Maßnahmen im Altbau betreffen in der Hauptsache umfassende Ertüchtigungsmaßnahmen wie den Brandschutz, die Schadstoffsanierung, die Haustechnik oder die Erneuerung von Oberflächen usw. In diesem Zuge werden unter Berücksichtigung der denkmalpflegerischen Belange Nutzungsänderungen von einzelnen Bereichen und Räumen erfolgen. Davon betroffen ist auch das Foyer, das auf Wunsch der Nutzer zukünftig mit Regalen und Leseplätzen ausgestattet werden soll. Weite Bereiche des Hauses, wie das Foyer, insbesondere der Lesesaal, haben sich aufgrund ihrer hohen baukünstlerischen Qualität im gemeinsamen Bewusstsein der Bürger von Stadt und Land verankert. Dies allein ist ein wichtiger Grund des weitgehenden Erhalts. Wir bewundern die Detailqualität, die den Innenausbau des Bestands auszeichnet. Bis "zur letzten Schraube" haben die Architekten des damaligen Staatlichen Bauamtes liebevoll Zeichnungen erarbeitet, im steten Bewusstsein, dass die Besucher die Architektur zuerst über das Detail erfassen.

\section{Dank}

Dass wir diese Qualität im Innenausbau des neuen Teils der Bibliothek beispielhaft übernehmen konnten, verdanken wir auch der guten Zusammenarbeit mit dem Amt Stuttgart von Vermögen und Bau Baden-Württemberg. Architektur gelingt nicht im stillen Kämmerlein. Sie beruht vielmehr auf einer kollegialen und konstruktiven Zusammenarbeit, für die wir uns ganz ausdrücklich bei Ilse Lange-Tiedje, der früheren Leiterin des Amtes, deren Nachfolger, Roland Wenk, und dessen Mitarbeiterinnen und Mitarbeitern bedanken.

Eine große Zahl an Fachingenieuren und -beratern war an der Planung unmittelbar beteiligt. Sie alle haben einen ganz wesentlichen Anteil an dem Projekt, für dessen erste Etappe wir für die Bibliothek, wie auch für die Stadt und das Land viel Erfolg wünschen.

Dr. Hannsjörg Kowark war es leider nicht vergönnt, die Fertigstellung zu erleben. Er hat sich für das neue Haus leidenschaftlich eingesetzt, ihm posthum und seiner Vertreterin, Martina Lüll, ganz großen Dank für ihr Vertrauen, auch ihren geduldigen Unterricht, die Funktionen und Aufgaben der Bibliothek betreffend. Dr. Rupert Schaab, der die Aufgabe mit den Mitarbeiterinnen und Mitarbeitern nun übernommen hat, ebenfalls herzlichen Dank, verbunden mit dem gemeinsamen Wunsch, nun den Altbau in derselben Intensität und Qualität fertigzustellen.

Arno Lederer Fotos: Brigida Gonzáles 